\title{
Estudio morfofuncional de la marcha humana
}

\author{
DANKLOFF C., RODRIGUEZ R., FERNANDEZ-VALENCIA R. \\ Dpto. de Ciencias Morfológicas y Cirugía. Facultad de Medicina. Universidad de Alcalá de Henares
}

\section{RESUMEN}

Se ha estudiado la marcha con dos plataformas de fuerzas (dinamométricas) en 60 sujetos, 30 varones y 30 mujeres, sin patología aparente del aparato locomotor, con edades comprendidas entre 17 y 22 años.

Presentamos los resultados obtenidos y establecemos a partir de los mismos, los valores medios normales de los parámetros más significativos de la marcha.

Con el fin de observar la existencia o no de una relación entre la morfología y las variables estudiadas en la marcha, se correlacionaron las magnitudes y los tiempos de las fuerzas desarrolladas en los tres ejes del espacio, con diversos parámetros antropométricos.

Los parámetros antropométricos que mayor correlación presentaron con los parámetros de marcha $(p$ $0,001)$ fueron el diámetro biacromial, el ancho maleolar dcho. con apoyo, y el ancho maleolar izquierdo.

\section{SUMMARY}

With two force platforms, we analyzed the gait of 60 students, 30 men and 30 women, aged betwen 17 and 22 , and we present here the obtained results. Starting from these results, we stablished the normal mean values of the most significative gait parameters.

In order to observe the existence or not of any relation between morphology and the considered gait variables, we correlated force magnitudes and times at which the forces were developed on the three space axes, with several anthropometric parameters.

The anthropometric parameters which presented the highest correlation coefficients with gait parameters were: biacromial diameter, right maleolar width \#with support\#, and left maleolar width.

Keywords: Gait, Ground reaction forces, Kinetics, Human walking, Morphologic Anthropology.

\section{INTRODUCCION}

La importancia que la actividad de la locomoción bipodal tiene para el desenvolvimiento normal del desplazamiento humano justifica plenamente el esfuerzo del análisis de marcha (Vera et al., 1985).

Se caracteriza la marcha por una repetición del patrón de movimientos corporales, existiendo en cada paso un pequeño intervalo de tiempo en que se encuentran los dos pies apoyados en el suelo. Entre cada dos dobles apoyos, un solo pie soporta el peso del cuerpo, a la vez que el otro se traslada de atrás hacia delante. Durante el período de contacto con la superficie de marcha el pie está relativamente estacionario, y al proporcionar el soporte del cuerpo existen fuerzas actuando entre el pie $y$ dicha superficie.

Las plataformas dinamométricas permiten registrar las fuerzas ejercidas por el cuerpo mediante los pies durante la fase de apoyo de la locomoción, así como establecer las curvas-tipo en función de la importancia de esas fuerzas y su distribución en el tiempo.

Si bien los patrones básicos de las fuerzas de reacción pie-suelo durante la locomoción han sido ya estudiados durante la primera mitad de este siglo (Elftman, 1939; Bresler \& Frankel, 1950), no han sido bien establecidas las diferencas entre varones y mujeres. Por ello hemos estudiado la marcha humana con dos plataformas dinamométricas en una población adulta, compuesta de varones y mujeres.

En el análisis de la marcha apenas ha sido estudiada la correlación existente entre parámetros morfológicos y parámetros obtenidos a partir de las plataformas de fuerzas. Solamente podemos mencionar algunas correlaciones realizadas con la longitud de paso o el tiempo de apoyo (Lord et al., 1977, Kirtley et al., 1985). Por ello otro de nuestros objetivos fue comprobar la relación entre varias dimensiones morfológicas y los parámetros de marcha.

\section{MATERIAL Y MÉTODO}

Se ha estudiado la marcha en 60 individuos, estudiantes de la Facultad de Medicina de la Universidad de Alcalá de Henares, 30 varones y 30 mujeres, con edades comprendidas entre 17 y 22 años, y sin presentar patología aparente del aparato locomotor.

El pasillo de marcha utilizado (fig. 1) está constituido por dos plataformas dinamométricas, tipo AHDA-IBV, que consisten en dos superficies metálicas de $45 * 60$ $\mathrm{cm}$ colocadas en el plano del suelo, y dispuestas de tal forma que no interfieren el ritmo de marcha.

Cada plataforma presenta 4 captadores de fuerzas, provistos de galgas extensiométricas orientadas en los tres planos del espacio, y cuya deformación es transmitida eléctricamente hasta un ordenador, siendo estas señales tratadas mediante el software adecuado. 
Los sujetos pasaban por el pasillo de marcha a lo que ellos consideraban su velocidad normal, registrándose el apoyo del pie derecho y del pie izquierdo, obteniéndose una representativa gráfica fuerza-tiempo (fig. 2), donde se analizan las fuerzas generadas al pasar sobre la superficie de las plataformas, expresándose la fuerza en newtons y el tiempo en ms. La línea horizontal que cruza la gráfica corresponde al peso del individuo, y las rectas verticales discontinuas indican el inicio y el final de cada apoyo así como el tiempo de doble apoyo.

En cad apoyo observamos las resultantes de las fuerzas verticales (las de mayor magnitud), anteroposteriores y lateromediales, estudiando 50 variables a partir del registro (Gómez Pellico et al., 1990): parámetros temporoespaciales, fuerzas de reacción pie-suelo máximas, tiempos de las fuerzas de reacción máximas, e índices temporales de las fuerzas de reacción máximas y del cruce.

Entre los parámetros temporoespaciales hemos considerado la longitud de paso (LPASO), distancia en $\mathrm{mm}$ entre el inicio del apoyo del talón derecho y el inicio del apoyo del talón izquierdo; el tiempo de paso (TPASO), duración en ms desde el inicio del apoyo del talón dcho. hasta el inicio del apoyo del talón izdo.; el tiempo de doble apoyo (TDA), tiempo transcurrido desde el inicio del apoyo del talón izdo., hasta el despegue del pie

Fig. 2. Gráfica fuerza - tiempo. Explicación en el texto.

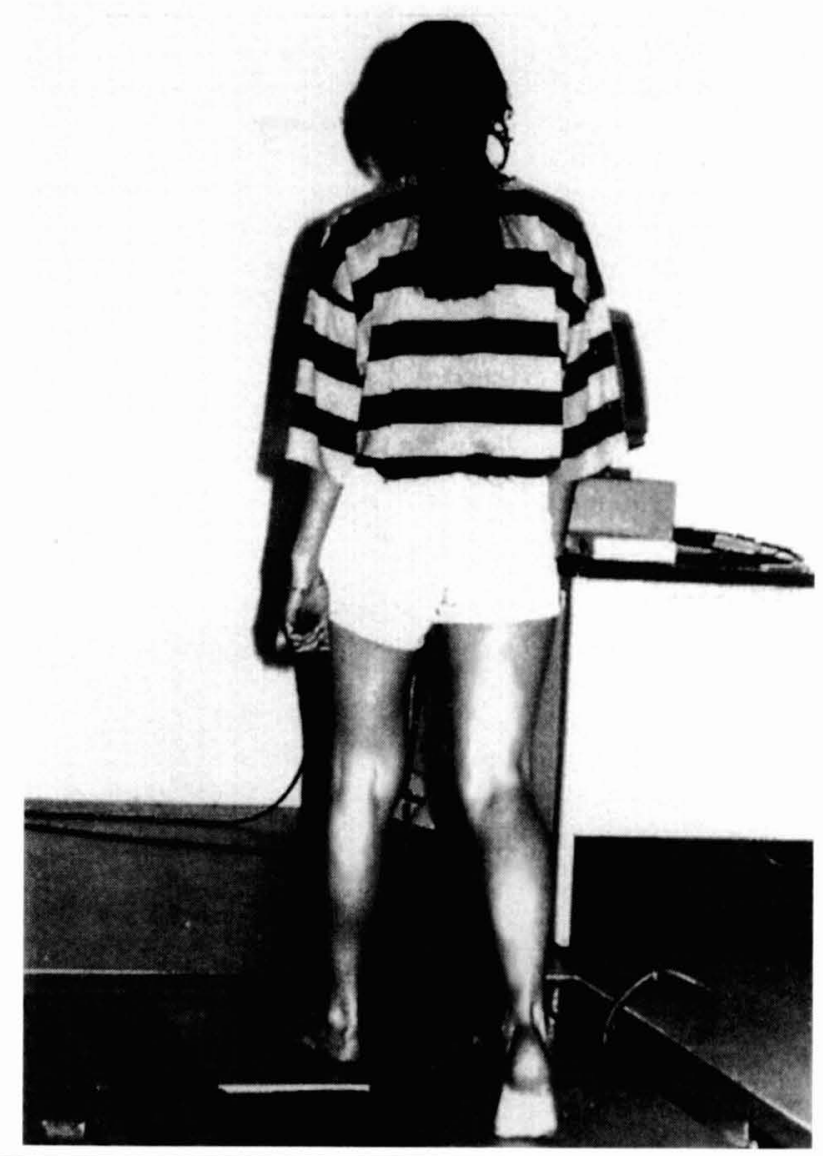

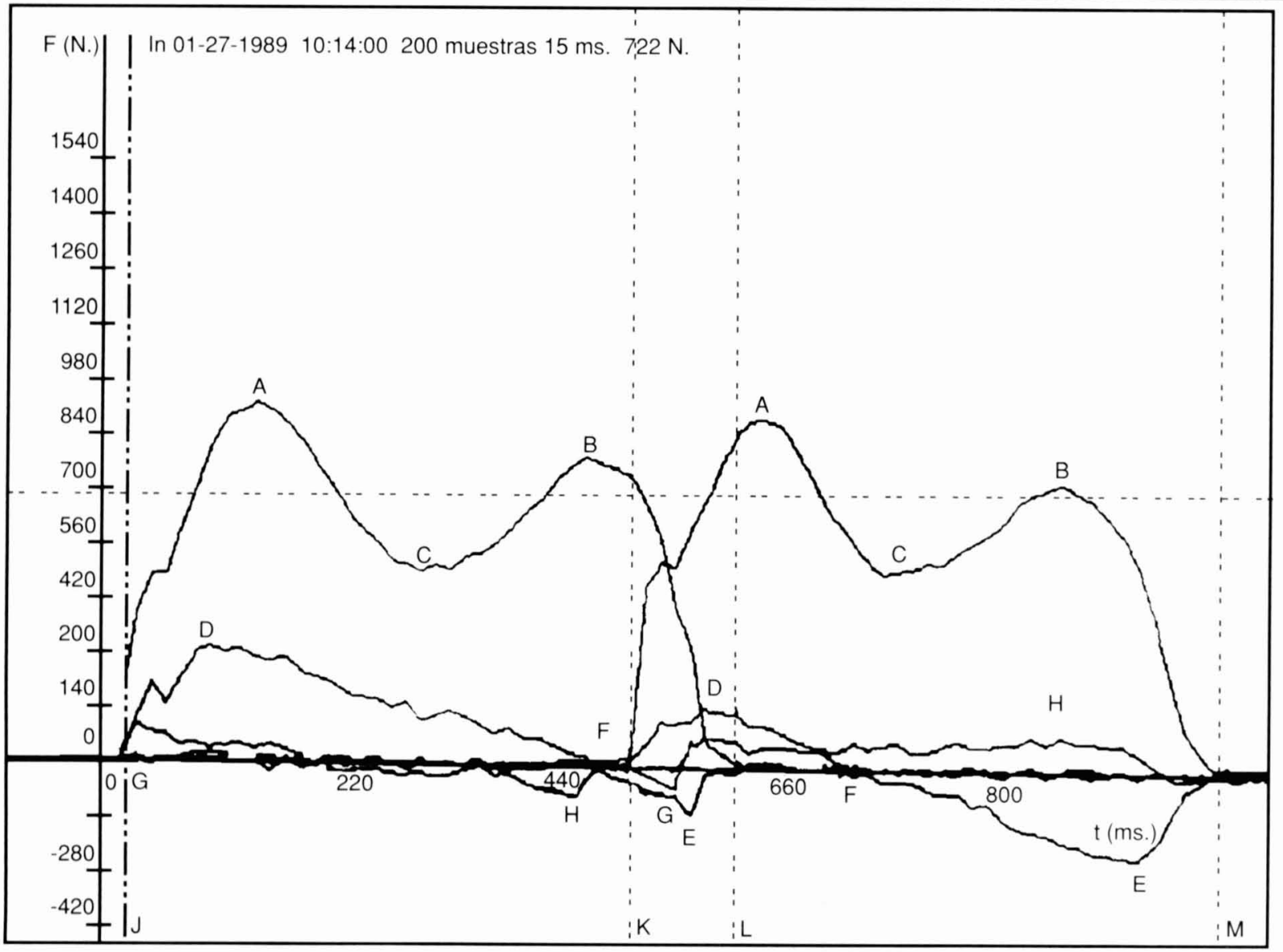


dcho.; el tiempo de apoyo, derecho e izdo. (TAD, TAI), es el tiempo en que el pie está en contacto con la plataforma. El coeficiente apoyo dcho.-apoyo izdo. (CADAI), es la relación temporal del apoyo dcho. e izdo., estudiando además los siguientes índices temporales, expresados en tanto por ciento: índice doble apoyoapoyo derecho (IDAAD), índice doble apoyo-apoyo izdo. (IDAAI), índice apoyo dcho.-apoyo total (IADAT), índice apoyo izdo.-apoyo total (IAIAT), e indice doble apoyo-apoyo total (IDAAT).

De los parámetros cinéticos, expresamos la magnitud de las fuerzas de reacción pie-suelo máximas, normalizadas con el peso corporal, y el tiempo en que suceden, en ms. La fuerza vertical del apoyo proximal (FVAP, fig. 2-A, A'), es el primer pico en la curva de las fuerzas verticales frente al tiempo; el segundo pico corresponde a la fuerza vertical del apoyo distal (FVAD, fig. 2$\left.\mathrm{B}, \mathrm{B}^{\prime}\right)$; el valor mínimo del valle formado entre ambas fuerzas verticales máximas, es la fuerza vertical mínima media (FVMIN, fig. 2-C, C'). La curva de las fuerzs anteroposteriores presenta dos cumbres inversas. La primera cumbre, positiva, cuya cima corresponde a la fuerza máxima anterior (FMA, fig. 2-D,D'), y la segunda cumbre, negativa, cuyo pico es la fuerza máxima posterior (FMP, fig. 2-E, $E^{\prime}$ ); el punto en que se invierte la cuva, i.e., las fuerzas pasan de ser anteriores a posteriores, o en otros términos, termina la frenada y comienza el despegue, es el punto neutro (TPN, fig. 2-F, F'). Debido a la pequeña dimensión de las fuerzas transversales, y a la gran variabilidad de las curvas, solamente referimos la magnitud de la fuerza máxima lateral (FML, fig. 2-H, $\mathrm{H}^{\prime}$ ), y de la fuerza máxima medial (FMM, fig. 2-G,G'). También valoramos los siguientes índices, que se obtuvieron mediante la relación entre el tiempo de cada una de las fuerzas máximas y el tiempo de apoyo de la extremidad, expresados todos en tanto por ciento: índice de la fuerza vertical del apoyo proximal (IFVAP), índice de la fuerza vertical del apoyo distal (IFVAD), índice del apoyo unipodal (IAU), que refiere el tiempo transcurrido entre ambas fuerzas verticales máximas al tiempo de apoyo, índice de la fuerza máxima anterior (IFMA), índice de la fuerza máxima posterior (IFMP), índice del punto neutro (IPN), y tres índices del cruce, que se refieren al momento en que coinciden las fuerzas verticales del apoyo dcho. e izdo. en la fase de doble apoyo, el índice de las fuerzas verticales en el cruce con respecto al tiempo de apoyo total (ICFVV), con respecto al tiempo de doble apoyo (ICDA), y el valor normalizado de las fuerzas verticales en el cruce (VNFFVC).

Además en cada individuo medimos 23 parámetros morfológicos, siguiendo las indicaciones de Cameron (1978) y Flügel et al. (1986), empleando material antropométrico homologado.

\section{RESULTADOS Y DISCUSION}

Presentamos los valores medios estimados para las 23 variables morfológicas (tabla 1) y las 50 variables de marcha normal (tabla 2), en varones y en mujeres.

En cuanto a los parámetros morfológicos, queremos destacar que todas las variables presentaron diferencia estadísticamente demostrable entre varones y mujeres, excepto los perímetros del muslo y los diámetros pélvicos, lo cual concuerda con los estudios de Carter et al. (1982), Piedade (1984) y Williams et al. (1987), en estudiantes y en corredores de élite.

Los parámetros de marcha que mostraron diferencia estadisticamente significativa entre varones y mujeres

TABLA I

PARAMETROS MORFOLOGICOS, VALORES MEDIOS ESTIMADOS

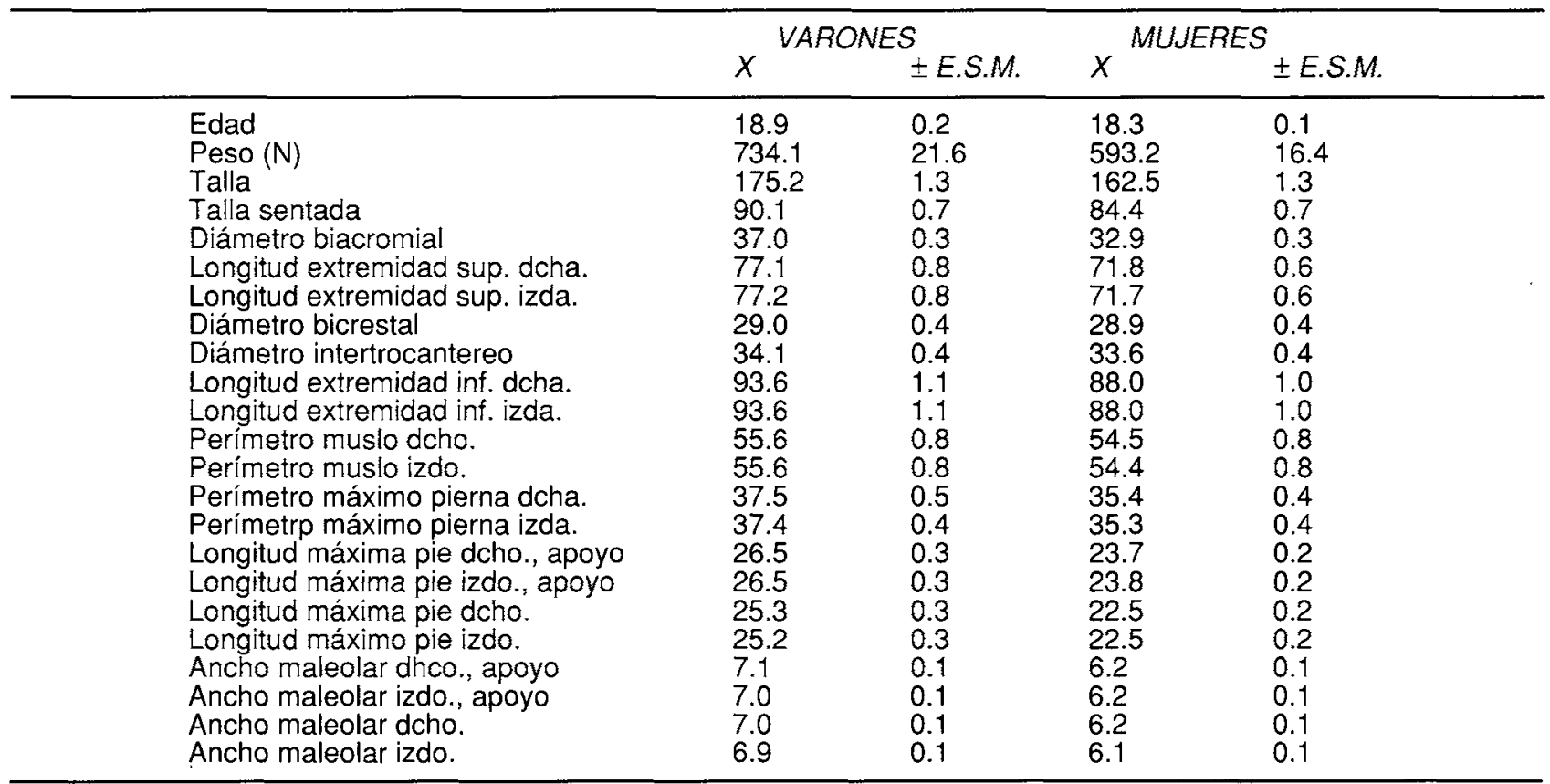


TABLA 2 MARCHA NORMAL, VALORES MEDIOS ESTIMADOS

\begin{tabular}{lllll}
\hline & \multicolumn{2}{l}{ VARONES } & \multicolumn{2}{l}{ MUJERES } \\
& $X$ & $\pm E . S . M$. & $X$ & \pm E.S. \\
\hline LPASO & 678.2 & 9.3 & 653.9 & 8.6 \\
TPASO & 599.5 & 11.6 & 570.0 & 11.4 \\
TDA & 143.0 & 5.3 & 127.0 & 4.8 \\
TAD & 739.5 & 15.5 & 695.5 & 14.6 \\
TAI & 773.0 & 15.6 & 721.0 & 12.0 \\
CADAI & 0.951 & 0.010 & 0.964 & 0.008 \\
IDAAD & 19.4 & 0.484 & 18.2 & 0.431 \\
IADAT & 53.5 & 0.430 & 53.9 & 0.305 \\
IAIAT & 56.4 & 0.241 & 55.9 & 0.218 \\
IDAAT & 10.4 & 0.257 & 9.8 & 0.271 \\
FMAD & 0.228 & 0.013 & 0.212 & 0.012 \\
FMPD & 0.176 & 0.008 & 0.181 & 0.007 \\
FMAI & 0.233 & 0.006 & 0.244 & 0.010 \\
FMPI & 0.169 & 0.006 & 0.162 & 0.005 \\
FVAPD & 1.094 & 0.010 & 1.060 & 0.012 \\
FVADD & 1.094 & 0.010 & 1.060 & 0.012 \\
FVMIND & 0.830 & 0.001 & 0.836 & 0.012 \\
FVAPI & 1.031 & 0.011 & 1.033 & 0.011 \\
FVADI & 1.031 & 0.011 & 1.049 & 0.009 \\
FVMINI & 0.832 & 0.007 & 0.834 & 0.009 \\
FMMD & 0.176 & 0.009 & 0.184 & 0.008 \\
FMLD & 0.016 & 0.005 & 0.009 & 0.002 \\
FMLI & 0.070 & 0.004 & 0.062 & 0.003 \\
FMMI & 0.051 & 0.005 & 0.056 & 0.005 \\
TFVAPD & 175.8 & 7.3 & 177.0 & 7.3 \\
TFVAPD & 561.3 & 12.3 & 517.5 & 10.9 \\
TFVAPI & 179.0 & 6.4 & 180.5 & 7.2 \\
TFVADI & 585.0 & 13.3 & 527.0 & 9.4 \\
TFMAD & 118.3 & 8.5 & 128.0 & 9.6 \\
TFMAI & 129.0 & 9.9 & 113.0 & 7.9 \\
TFMPD & 650.0 & 12.9 & 613.5 & 14.2 \\
TFMPI & 679.5 & 14.1 & 625.0 & 12.3 \\
TPND & 526.0 & 17.1 & 489.3 & 17.9 \\
TPNI & 514.0 & 16.2 & 505.0 & 14.7 \\
IPND & 71.4 & 1.8 & 70.1 & 1.8 \\
IPNI & 66.4 & 1.3 & 69.8 & 1.2 \\
IFMAD & 16.0 & 1.1 & 18.1 & 1.2 \\
IFMAI & 16.4 & 1.1 & 15.6 & 1.0 \\
IFMPD & 88.0 & 0.5 & 88.1 & 0.4 \\
IFMPI & 87.9 & 0.5 & 86.6 & 0.7 \\
IFVAPD & 23.6 & 0.7 & 25.3 & 0.7 \\
IFVADD & 75.9 & 0.5 & 74.4 & 0.5 \\
IFVAPI & 23.1 & 0.5 & 24.9 & 0.8 \\
IFVADI & 75.6 & 0.7 & 73.1 & 0.7 \\
IAUD & 52.3 & 0.9 & 49.2 & 0.9 \\
IAUI & 52.5 & 0.6 & 48.3 & 1.0 \\
ICFFV & 48.3 & 0.3 & 48.1 & 0.3 \\
ICDA & 45.4 & 1.7 & 40.3 & 1.8 \\
VNFFVC & 62.2 & 1.5 & 62.2 & 1.3 \\
\hline & & & & \\
\hline
\end{tabular}

TABLA 3.

COEFICIENTES DE CORRELACION ( $p$ 0.001), $Y$ ECUACIONES DE LAS RECTAS DE REGRESION $(n=60)$

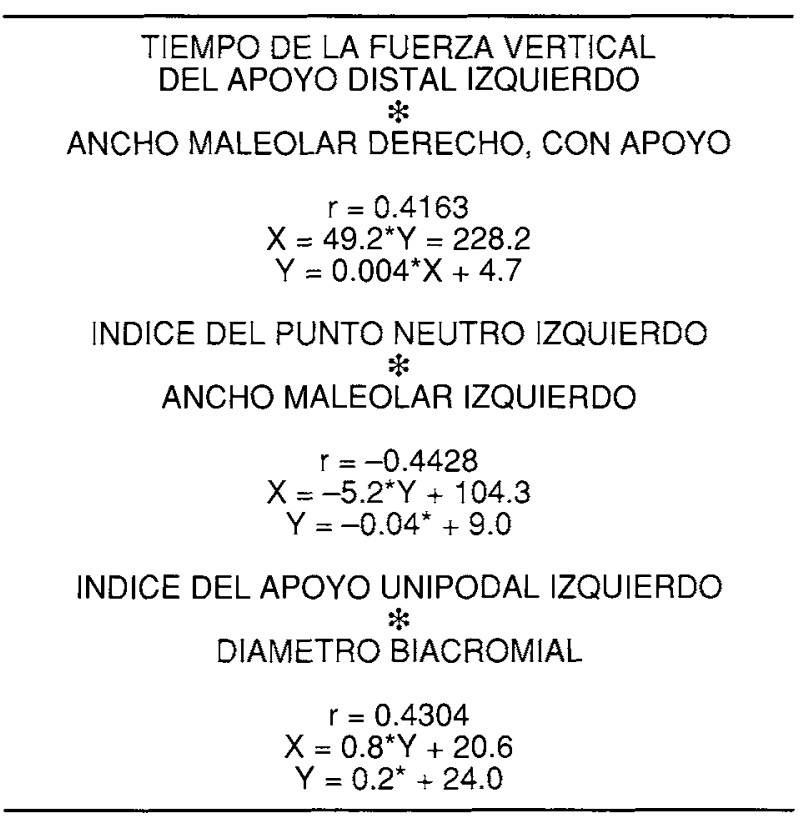

fueron los tiempos de doble apoyo $(\mathrm{p} \leq .05)$, de apoyo dcho. ( $\mathrm{p} \leq .05)$ y de apoyo izdo. ( $\mathrm{p} \leq .01$ ), mayores en varones, al igual que la fuerza vertical del apoyo distal dcho. e izdo., que suceden más tardíamente en varones que en mujeres, tanto si lo expresamos en forma de ms como en forma de índices (TFVADD, $\mathrm{p} \leq .01$; TFVADI, $\mathrm{p} \leq .001 ;$ IFVADD, $\mathrm{p} \leq .05 ;$ IFVADI, $\mathrm{p} \leq .01$; IAUD, $\mathrm{p} \leq .05 ;$ IAUI, $\mathrm{p} \leq .001)$. También mostraron diferencias el tiempo de la fuerza max. post. izda. ( $\mathrm{p} \leq$ $.01)$ y el índice del cruce de las fuerzas verticales con respecto al doble apoyo $(\mathrm{p} \leq .05)$.

De los resultados del estudio de la correlación entre los parámetros morfológicos y los parámetros de marcha indicamos los índices de correlación más significativos ( $p .001$, tabla 3 ) y las correspondientes ecuaciones de regresion.

\section{CONCLUSIONES}

El análisis de la marcha humna con plataformas dinamométricas permite establecer patrones de marcha normales que dan las características antropológicas de la locomoción tanto cinéticas como temporales.

Se han obtenido los valores medios estimados de la población de 50 parámetros cinéticos y temporoespaciales de la marcha humana normal analizada por plataformas dinamométricas.

Los parámetros de la marcha que mostraron ser significativamente mayores en el vaón que en la mujer fueron el TDA, el TAD, el TAI, el TFVADD, el TFVADI, el TFMPI, el IFVADD, el IFVADI, el IAUD, el IAUI, y el ICDA.

Presentamos los índices de correlación más significativos ( $\mathrm{p} .001)$ entre los parámetros de marcha y los morfológicos (tabla 3). 


\section{BIBLIOGRAFIA}

Bresler, B., Frankel, J.P. The forces and moments in the leg during level walking. Trans Am Soc Mech Engrs 1950; 72: 25-36.

Cameron, N. The method of auxological anthropometry Human Growth 2. New York: Plenum Press, F. Falkner \& J.M. Tanner, 1978

Carter, J.E.L., Ross, W.D., Aubry, S.P., Hebbelinck, M., Borms, J. Anthropometry of Montreal Olympic Athletes. Medicine Sport 1982; 16: 25-52.

Elftman, $\mathrm{H}$. Forces and Energy Changes in the Legs during Walking. Am J of Physiol 1939; 125: 339-356.

Flügel, B., Greil, H., Sommer, K. Antropologischer Atlas, Grundlagen un daten. Berlin, DDR, Verlag Tribune, 1986.

Gómez Pellico, L., Forriol Campos, F., Dankloff, C. Estudio cinético de la marcha normal. Rev Orthop Traum 1990; 34IB(6): 699-703.
Kirtley, C., Whittle, M.W., Jefferson, R.J. Influence of walking speed on gait parameters. J Biomed Eng, 1985; 7: 282288.

Lord, G., Gentaz, R., Gandolfi, R. La marche normale et ses altérations après arthroplastie totale au membre inférieur. Revue de Chirurgie Orthopédique 1977; 63: 221-236.

Piedade, A.J. Contribution to the study of sex differences during growth of portuguese students (Queluz town). In Human growth and development, Belgium: J. Borms, A. Sand, C. Susanne, M. Hebbelinck (eds), 1984.

Vera, P., Hoyos, J.V., Nieto, J. Biomecánica del Aparato locomotor. Valencia: Instituto de Biomecánica de Valencia, 1985.

Williams, K.R., Cavanagh, P.R., Ziff, J.L. Biomechanical studieds of elite female distance runners. Int J Sports Med 1987; 8: 107-108 (supl). 